\title{
Operaciones Unitarias: Enfocadas a los evaporadores en la elaboración de productos de consumo humano.
}

\author{
Operaciones Unitarias: Enfocadas a los evaporadores en la elaboración de \\ productos de consumo humano.
}

Sayuri Monserrath Bonilla Novillo ${ }^{1}$, Jaime Iván Acosta Velarde ${ }^{2}$, Gloria Elizabeth Miño Cascante ${ }^{3}$ Andrés Joao Noguera Cundar ${ }^{4}$ Mónica Alexandra Moreno Barriga ${ }^{5}$

Recibido: 20-02-2019 / Revisado: 28-02-2019 /Aceptado: 04-03-2019/ Publicado: 05-04-2019

\begin{abstract}
.
DOI: https://doi.org/10.33262/cienciadigital.v3i2.451

The present work shows an analysis of the evaporators used in the elaboration of products for human consumption, thus we have the multiple effect evaporators $(\mathrm{EME})$ that are used in the food industry for the production of vegetable oils, dairy products, micelles distillation of food since their products are of high quality; the key to its effectiveness is in the control of the evaporation variables that they provide, since it is constituted by a series of bodies called effects which have a simple but very effective operating principle until the completion of their work. In the alcoholic beverage industry, evaporators are fundamental, since their production process depends on the type of distillation applied, which is done in evaporators significantly smaller than the EME and its treatment is given a higher heating rate, the evaporator used consists of a vertical tube usually made of glass or surgical stainless steel accompanied by a Graham condenser that is subjected to three different heating speeds to conserve the calorific gradient, the variety, quality and

1 Escuela Superior Politécnica de Chimborazo, Facultad de Administración de Administración de Empresas, Riobamba, Ecuador, smbonilla@espoch.edu.ec

2 Escuela Superior Politécnica de Chimborazo, Facultad de Mecánica, Riobamba, Ecuador, ji_acosta@espoch.edu.ec

3 Escuela Superior Politécnica de Chimborazo, Facultad de Mecánica, Riobamba, Ecuador, gmino@espoch.edu.ec

4 Escuela Superior Politécnica de Chimborazo, Facultad de Mecánica, Riobamba, Ecuador, andres.noguera@espoch.edu.ec

5 Escuela Superior Politécnica de Chimborazo, Facultad de Mecánica, Riobamba, Ecuador, monica.moreno@espoch.edu.ec
\end{abstract}


purity of the liquor depends on the heating time and the proportions of the different raw materials.

Keywords: Evaporators - Production - Consumer products - Food industry.

\section{Resumen.}

El presente trabajo muestra un análisis de los evaporadores utilizados en la elaboración de productos de consumo humano, así se tiene los evaporadores de múltiple efecto (EME) que son utilizados en la industria alimenticia para la producción de aceites vegetales, lácteos, destilación de micelas de alimentos ya que sus productos obtenidos son de gran calidad; la clave de su efectividad está en el control de las variables de evaporación que brindan, pues se encuentra constituido por una serie de cuerpos llamados efectos los cuales tienen un principio de funcionamiento sencillo pero muy efectivo al momento de realizar su trabajo. En la industria de bebidas alcohólicas los evaporadores son fundamentales, pues su proceso de producción depende del tipo de destilación aplicada, el cual se lo hace en evaporadores significativamente más pequeños que los EME y su tratamiento viene dado a mayor velocidad de calentamiento, el evaporador utilizado consta de un tubo vertical generalmente de vidrio o de acero inoxidable quirúrgico acompañado de un condensador de Graham que es sometido a tres velocidades diferentes de calentamiento para conservar el gradiente calorífico, la variedad, calidad y pureza del licor depende del tiempo de calentamiento $\mathrm{y}$ de las proporciones de las diferentes materias primas.

Palabras claves: Evaporadores - Producción - Productos de consumo - Industria alimenticia.

\section{Introducción.}

Muchas operaciones unitarias utilizadas en la elaboración de alimentos requieren de transferencia de calor, desde o hacia estos. "En determinados procesos de la industria alimentaria, la transmisión de calor adquiere una importancia relevante en procesos tales como los diferentes tratamientos para la destrucción de microorganismos (esterilización, pasteurización, escaldado, entre otros) y conservación de alimentos mediante el frío (refrigeración y congelación), a la vez que resulta especialmente importante sobre las propiedades de los alimentos (color, olor, sabor, textura y valor nutritivo). La evaporación es una operación unitaria consistente en la separación de una mezcla líquida, generando a partir de la misma, por ebullición, un vapor integrado por los componentes más volátiles de aquella. Se utiliza para concentrar disoluciones obteniéndose vapor del disolvente". (Rodríguez, 1999). 
Es una operación muy empleada en diversas industrias, ya sea para tomar provecho de la concentración de la disolución (por ejemplo la concentración de la leche), o para aprovechar el disolvente evaporado (por ejemplo la obtención de agua desalinizada por ebullición del agua del mar y posterior condensación del vapor de agua). Normalmente se tiene varios evaporadores combinados, en los que se emplea el vapor generado en un evaporador como medio de calefacción del siguiente (se denominan evaporadores de múltiple efecto).

Para la producción de la panela utilizando un evaporador de múltiple efecto (EME) el objetivo es de mejorar los tiempos de procesos y la optimización de recursos, este tipo de evaporador trabajan con los vapores teniendo en cuenta las temperaturas de ebullición y transferencia de calor, mientras los vapores deben reducir el porcentaje de agua.

El funcionamiento de los EME, consiste en un funcionamiento por etapa llegando a las últimas fases, donde el vapor producido es enviado a un condensador, estos tipos de evaporadores son eficientes en término de tiempo y optimización de recursos. (Ordoñez, Hernández, \& Pedraza, 2012)

Los EME son utilizados también en la concentración de evaporación del jugo natural de naranja utilizando un evaporador de tres efectos, extraen el juego con un exprimidor eléctrico, aquí escogen muestras concentradas y sin concentrar, para un estudio de densidades, en los resultados del proceso de concentración se observaron los valores alojado de cada efecto realizado, donde el evaporador de doble efecto fue el de mayor concentración (Avalo, Pérez, \& Tovar, 2009).

Se considera una mezcla perfecta en la fase liquida, equilibrio de fases entre el vapor formado y la solución en ebullición. "Se efectuó el balance de masa global tomando el volumen de control de doble efecto y despreciando la masa de vapor retenida por ser mayor el volumen de líquido acumulado en cada efecto". (Avalo \& Alfredo, 2008).

En lo que respecta a la industria de bebidas alcohólicas se tiene que para la obtención del guarapo deshidratado, se realiza la concentración de este proceso en un evaporador al vacío mediante temperaturas establecida de entrada y de salida (Armando Díaz, 2015)

Para la elaboración de piloncillo se obtiene de la evaporación del jugo de caña hasta llegar a cristalización de la sacarosa para elaborar este producto se dividió en 3 secciones: 1. preparación de la caña de azúcar y extracción de jugo, 2. consiste en el clarificado y concentración del jugo y 3. Es el moldeo del piloncillo. Se cuenta con dos evaporadores de doble efecto en el equipo de concentración, el funcionamiento del mismo trata de que con el vapor generado por el primer evaporador se calienta el segundo. "Una vez concentrado el jugo es descargado en un tanque receptor, el cual se descarga a un evaporador atmosférico, donde se termina de concentrar a una temperatura y el jugo llega a la concentración. La 
calidad de este de producto se basa en la concentración del piloncillo" (Josué R. SolísPacheco, 2006)

En otro sector de la industria alimenticia los EME ayudan a la retención de nutrientes en la guayaba y feijoa, utilizando los mismos al vacío y a presión, con el fin de retener la vitamina $\mathrm{C}$ y los minerales. Este producto es conocido como bocadillo, la temperatura juega un papel muy importante al utilizar este tipo de máquina, esta temperatura incide en la permanencia del ácido ascórbico en los alimentos procesados en alta presión hidrostática. (Hernandez \& Borray, 2008).

Los evaporadores de multiefecto se destacan no solo en la producción de panelas y mieles sino que son muy utilizados en la industria alimenticia en general ya que realizan un proceso de evaporación abierta con lo cual se obtiene un ahorro energético durante la evaporación puesto que el proceso en si genera energía y este evaporador aprovecha dicha energía producida por el mismo proceso.

\section{Metodología y Desarrollo.}

Los evaporadores forman parte primordial en la elaboración de diferentes tipos de productos ya que gracias a su gran utilidad y ayuda al momento de realizar cada una de sus tareas específicas lo hacen de una manera efectiva y con los resultados requeridos por cada una de las industrias existentes.

"Un evaporador múltiple efecto (EME) es un equipo utilizado en la industria alimenticia, compuesto de varios cuerpos llamados efectos. En el primero, se suministra calor de una fuente exterior y se opera a una temperatura de ebullición alta establecida por la presión del sistema". (Forero, Bernal, \& Guerrero, 2014)

Los EME son utilizados ampliamente en la industria dedicada a la elaboración de pasta de tomate ya que la obtención de la misma se la hace mediante la concentración de sus solidos solubles, esta concentración se la hace generalmente en equipos al vacío, como los evaporadores de múltiple efecto, donde se controlan las variables del proceso para conseguir que el producto elaborado tenga mejores características. (Quintana, Maya, Medrano, \& Montes, 2015).

Otro sector que utiliza ampliamente este tipo de evaporadores multiefecto son las industrias de panelas las cuales cabe recalcar que las encontramos en mayor parte en Sudamérica "En la industria de la panela (denominada azúcar sin centrifugar por la FAO), se estima que los evaporadores de múltiple efecto (EME), permiten un mayor control e incrementan en un $36 \%$ la eficiencia global del proceso no obstante, la implementación de estas tecnologías requiere que, además del ajuste técnico del proceso, también se mantenga la calidad de la panela obtenida en los sistemas de evaporación abierta”. (Forero, Bernal, \& Guerrero, 2014). 
Los evaporadores en la industria no solo ofrecen servicios en la producción de un producto sino que también brindan servicios de limpieza de maquinarias utilizadas en la elaboración de productos alimenticios, ya que al terminar el ciclo de elaboración de un determinado producto termina con impurezas y sucias, cuando esto sucede se utiliza un cierto tipo de evaporador que gracias a su fuerte presión de vapor y gran elevación de calor ofrece una limpieza a fondo del 99,99\% en la eliminación de impurezas y suciedad. (Diaz, Gonzales, \& Alvarado, 2017).

Los sistemas de vaporizadores, que usan concentradores solares tipo Fresnel y trabajan en paralelo alcanzan temperaturas de trabajo de hasta $90{ }^{\circ} \mathrm{C}$ debido a las variaciones de temperatura y radiación por calentamiento solar los valores esperados de la producción no son los ideales, en este trabajo el vapor ingresa directamente a la bandeja inferior a través de un tubo al que se le han realizado pequeños orificios por donde escapa el vapor, de esta manera el destilado producido en la primera etapa se debe exclusivamente al vapor proveniente de los vaporizadores, en los cuales al momento de usar los concentradores solares tipo Fresnel, la producción de vapor es de aproximadamente $2 \mathrm{lt} / \mathrm{h}$, de manera que con el caudal ensayado prácticamente se trabaja con la mitad de vapor, es por ello que si se pretende aprovechar este vapor ingresado en su totalidad es necesario duplicar el área del destilador. (Esteban \& Franco, 2002)

La fase de pasteurización se realiza haciendo burbujear el vapor de agua a "baño María" dentro del recipiente pasteurizador. El vapor se condensa y transfiere el calor de condensación al agua elevando su temperatura. Como el recipiente está aislado las pérdidas de calor en el recipiente pasteurizador son pequeñas. La manguera de conexión entre el vaporizador y el recipiente pasteurizador por la que circula el vapor se envuelve con una manta aislante para evitar las pérdidas. Alcanzan $65^{\circ} \mathrm{C}$.

A partir de este momento se comienza a medir la temperatura en forma manual con un termómetro digital de termocupla. En un ensayo experimental se midieron las temperaturas en el "baño María" y en el interior de la olla que contenía 8 litros de agua. Como el "baño María" contenía 7 litros de agua la cantidad total de líquido dentro del pasteurizador era de 15 litros al inicio. Una vez que comienza la producción de vapor el aumento de temperatura es constante y varía aproximadamente $3{ }^{\circ} \mathrm{C}$ cada 5 minutos. Durante los primeros 30 minutos, se produce una disminución en la producción del vapor. Esta disminución de producción se debía a las características constructivas del vaporizador. Los dos litros de agua colman la capacidad del vaporizador. La radiación solar se concentra en la parte superior calentando rápidamente el agua a ese nivel. Cuando baja el nivel del agua a la altura de la aislación, la radiación no llega directamente a la misma calentándola por conducción del calor por las paredes de la caldera. Para mantener constante la producción de vapor se hace necesario cambiar la geometría del vaporizador de manera tal que todo el recipiente quede expuesto a la radiación. (Caso, Franco, Fernandez, Javi , \& Saravia, 2004)

En un proceso de separación de refinamiento de petróleo se calienta la base del elevador, el catalizador regenerado y con un alto contenido de energía, se pone en contacto directo con la alimentación líquida, previamente atomizada, para llevarla hasta su punto de ebullición y, posteriormente, hasta la vaporización. Una vez que la alimentación se encuentra en fase vapor, ésta se calienta hasta alcanzar la temperatura de mezclado. La etapa de vaporización 
se considera como un proceso instantáneo en ausencia de las reacciones de desintegración catalítica. Por lo anterior, la vaporización se modela en estado estacionario con la suposición de mezclado perfecto sin reacción. El vapor que se inyecta en esta zona, cuyo papel es favorecer el contacto de la alimentación con los sitios ácidos del catalizador, es tomado en cuenta en el balance de energía. La Ilustración 1 muestra esquemáticamente las entalpías de la alimentación, vapor de agua y el catalizador en la base del elevador antes y después de la vaporización. (Flores, 2006, pág. 46)

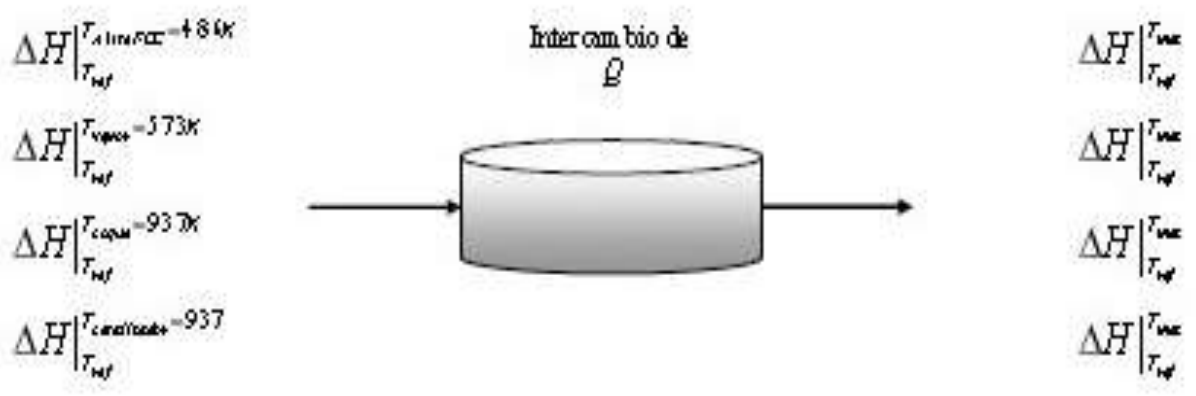

Figura 1. Esquema de entalpias.

Fuente: (Barajas \& Roman, 2014)

En el proceso de secado de alimentos los productos ingresan al secadero de alimento en base seca, a una determinada temperatura con un cierto grado de humedad. La temperatura y humedad del alimento a la salida de la cámara de enfriamiento ha cambiado. El caudal de aire húmedo para el secado tiene su temperatura determinada y una humedad absoluta. El aire atraviesa el alimento que se encuentra sobre la cinta transportadora. El aire se calienta en un calentador, con una determinada cantidad de calor entregada. Parte del aire del proceso, es reciclado y mezclado con aire atmosférico a una temperatura y una humedad específica. (Montesano \& Dipietro, 2013)

En la transmisión de calor de los evaporadores se incluyen las diferentes variables de cada una de las corrientes. En la cámara de condensación se alimenta una corriente de vapor que posee una determinada temperatura. El vapor condensa, y el único calor que cede es el de condensación, por lo que sale una corriente de agua líquida a la temperatura de condensación. El calor de condensación es transferido a través del área de intercambio del evaporador, y es captado por la corriente del alimento en la cámara de evaporación. En la cámara de evaporación se alimenta una corriente, que se halla a una temperatura, existiendo una entalpia, debido al calor que cede el vapor condensado. Es importante resaltar que las temperaturas de las corrientes de concentrado y de vapor que abandonan la cámara de evaporación son iguales, y se corresponden a la temperatura de ebullición de la disolución concentrada que abandona esta cámara. (Ibarz \& Barbosa, 2005)

Los evaporadores son utilizados también en la elaboración de helado ya que "Para desarrollar la evaluación energética del ciclo de refrigeración para la elaboración de helado fue necesario determinar los flujos de calor transferidos en los evaporadores. Esta mezcla húmeda una vez que se encuentra en los evaporadores antes mencionados intercambia calor ocurriendo propiamente el proceso de refrigeración a expensas de la evaporación del refrigerante en la zona de alta presión. Estos evaporadores alcanzan el estado de vapor saturado al extraerle el 
calor a la mezcla y al helado ocurriendo el propio proceso de refrigeración a expensas de la evaporación del refrigerante en la zona de baja presión". (MSc. Yanet Sariego-ToledoI, 2014).

Para finalizar la revisión de industrias en las que se utilizan los evaporadores nos vamos a referir a la producción de zumo de frutas especialmente el de naranja y nos dice que "El proceso de co-cristalización implica la concentración de jarabes de sacarosa por evaporación a temperatura elevada hasta que se consigue la cristalización espontánea de sacarosa. Cocristalización al zumo de naranja agria se le ajusto empleando Hidróxido de Calcio. Luego el zumo se llevó a un rota-evaporador, con el fin de concentrar el zumo hasta la concentración de solidos solubles a evaluar. El zumo concentrado fue etiquetado y almacenado en refrigeración”. (Ricardo D. Andrade, 2016)

\section{Discusión.}

Al comparar los evaporadores en las distintas ramas de la industria en general se tiene que los evaporadores de múltiple efecto o multiefecto (EME) forman parte primordial en la elaboración de los distintos productos en la industria alimenticia así como también en la producción de aceites vegetales, lácteos, destilación de micelas de alimentos siento esta la materia prima de varios productos de consumo humano como son jabones de baño, cremas, en si productos de limpieza personal. Ya que la calidad de los productos finales que estos equipos ofrecen es muy buena, además que los EME son los más utilizados en empresas alimenticias desde su aparición a mediados del siglo XX.

La clave de su efectividad está en el control de las variables de evaporación que nos brindan estos evaporadores ya que básicamente el EME es una serie de cuerpos llamados efectos estos tienen un principio de funcionamiento sencillo pero muy efectivo al momento de realizar su trabajo ya que al primer cuerpo se le introduce temperaturas altas de ebullición pero de manera externa al momento de pasar al segundo cuerpo la temperatura de vapor del anterior cuerpo sirve como medio de calentamiento a una temperatura inferior así que cada proceso tiene una presión de vapor inferior a la anterior esto ayuda a mantener el sistema sobrecalentado manteniendo así el gradiente de calor en el sistema pero constante gracias a este efecto de vacío las características de los productos son buenas, dependiendo del trato que tengan los distintos productos alimenticios se necesita de un EME de dos, tres o los cuerpos que sean necesarios para obtener los mejores resultados.

En la industria alimenticia la solución ideal del punto de ebullición del vapor en los alimentos parte de la ley de Raoult la cual ofrece un correcto proceso de elaboración de los distintos productos, dicha ley se presenta a continuación:

$$
\Delta T_{B}=T-T_{W}=\frac{-T_{W}}{1+\left(\frac{\Delta H_{V}}{R T_{W} \operatorname{Ln}\left(X_{W}\right)}\right)}
$$




\section{Dónde:}

$\Delta T_{B}$ Es el aumento del punto de ebullición.

$T$ Es el punto de ebullición de la solución.

$T_{W}$ Es el punto de ebullición del agua pura.

$\Delta H_{V}$ Es el calor latente de evaporación del agua.

$R$ Es la constante de los gases ideales.

$X_{W}$ Es la fracción molar del agua.

Por otro lado está la industria de bebidas alcohólicas en la cual los evaporadores forman parte primordial en el proceso de las mismas, ya que en si todo el proceso de producción de una bebida alcohólica depende del tipo de destilación que se aplique cuyo proceso se lo hace en evaporadores significativamente más pequeños que los EME ya que el volumen de materia prima que se introduce en ellos es en menor cantidad y el tratamiento de la misma viene dada a mayor velocidad de calentamiento lo cual se consigue en un recipiente este caso un evaporador, más pequeño y cerrado, los cuales consisten de un tubo vertical generalmente de vidrio o de acero inoxidable quirúrgico acompañado de un condensador de Graham este evaporador es sometido a tres velocidades diferentes de calentamiento para conservar el gradiente calorífico, al momento en que el producto empieza a entrar a su punto de fusión es decir que sufre un cambio de estado líquido a gas entra en acción el condensador enfriando el fluido drásticamente y gracias a un sistema de destilación integrado en el evaporador obtenemos el licor perfectamente destilado y de una alta calidad teniendo siempre en cuenta que el tiempo de calentamiento de las diferentes materias primas para la gran variedad de licores que encontramos en el mercado depende de que tan puro y fino sea el mismo y dichos tiempos y proporciones de materia prima se tiene dadas en tablas y manuales.

Se resalta algo interesante también que realizan los evaporadores ya que no son utilizados netamente en la elaboración de algún tipo de producto de consumo humano, sino que también en la limpieza de otros equipos de producción alimenticia como por ejemplo los contenedores de productos lácteos al finalizar su operación quedan totalmente manchados del producto que estaba en elaboración y al tratarse de un producto de tratamiento delicado todos sus equipos y utensilios deben estar en perfecto estado y limpieza nítida y es aquí en donde intervienen un tipo de evaporadores dedicados a la limpieza y desinfectación de equipos, gracias a sus altas presiones de vapor y elevadas temperaturas la limpieza llega a un punto del 99,99\% de limpieza al acabar su operación siendo así los evaporadores elementos de suma importancia en todo el aspecto industrial a nivel mundial.

En la pasteurización de lácteos los evaporadores EME juegan un papel muy importante ya que este tipo de industrias se dedican a la elaboración de productos que forman parte de la dieta diaria del ser humano, para esto se debe tener cuenta todos los parámetros de 
aislamiento y tener un amplio conocimiento de transferencia de calor. La ergometría de los sistemas de evaporación determinara un proceso de calentamiento rápido y uniforme.

"La vaporización también se utiliza en la producción de FCC (Craqueo Catalítico Fluido). Se llega a un proceso de vaporización cuando en la base de un riser donde se encuentra almacenado un líquido se pone en contacto con el catalizador el mismo que se encuentra cargado de energía, llevando así al líquido a su punto de ebullición y posteriormente a la evaporación. La etapa de vaporización se considera como un proceso instantáneo en ausencia de las reacciones de desintegración catalítica". (Buchanan, 1994; Theologos y Markatos, 1999; Mirgain et al., 2000). Analizando el proceso anterior una vez que se produce el vapor este tiene un contacto con las zonas acidas del catalizador. En este punto se realiza un balance de energías donde se puede estudiar las Entalpias en el riser. Para evaluar la entalpia de la alimentación del trabajo realizado en el proceso FCC es importante tomar en cuenta los efectos térmicos y los efectos de presión. Se utiliza la termodinámica continua para evaluar los efectos de presión mientras que se utiliza el (calor específico) para evaluar las entalpias del vapor de agua, coque y catalizador.

Los evaporadores a nivel industrial son muy utilizados en sus distintas áreas e industrias, pero específicamente en el área alimenticia se destacan mucho más que en otros sectores empresariales siendo los de mayor relevancia los evaporadores multiefecto (EME) ya que bridan calidad en la elaboración de sus distintos productos conservando las características sobresalientes de los mismos.

\section{Conclusiones.}

- Los evaporadores de multiefecto (EME) son los más utilizados en lo que respecta a la industria alimenticia gracias a que ofrece una presión de vapor al vacío y con un calor gradiente, esto da un efecto beneficioso en los alimentos ya que conserva perfectamente sus características más importantes.

- Los EME son más eficientes en término de tiempo y optimización de recursos ya que para una empresa su finalidad es producir a menor costo y tiempo.

- Los EME son los más utilizados en la concentración de nutrientes ya que brinda mejores resultados en la retención de nutrientes debido a sus múltiples fases.

- Los sistemas de evaporación tienen un principio de funcionamiento en base a parámetros termodinámicos, el aislamiento y la transferencia de calor cumplen un rol importante en su diseño.

- Los procesos de evaporación, se utilizan en la obtención de hidrocarburos en los que se puede separar los distintos derivados del petróleo, ya que al elevar la temperatura y al poner en contacto con un fluido se da una serie de entalpias teniendo una evaporación del material liviano lo que produce la separación del combustible del coque. 


\section{Referencias bibliográficas.}

Andrade, R. D., Blanquicett, K., \& Rangel, R. D. (2016). Efecto del pH, Sólidos Solubles y Zumo Adicionado sob re el color y la Vitamina $C$ de Zumo de Naranja Agria Cocristalizado. Scielo, 27(6), 129-134.

Andrade, R. D., Lemus, R., \& Pérez, C. E. (2011). Modelos de isotermas de sorcion para alimentos: usos y limitaciones. Scopus, 18(3), 325-334.

Armando Díaz, F. C. (24 de 03 de 2015). Evaluación de los parámetros de secado para la obtención de guarapo deshidratado empleando un. REDALYC.ORG.

Avalo, B., \& Alfredo, V. (12 de 08 de 2008). Modelado y simulación de un evaporador de triple efecto para la concentración de jugos naturales. Scielo, 11.

Avalo, B., Pérez, S., \& Tovar, M. (06 de 11 de 2009). Caracterización preliminar del proceso de concentración del jugo natural de naranja en un evaporador de tres efectos. Sciencedirect, 8.

Ávila-de Hernández, R. (2016). La concentración de jugos de fruta: Aspectos básicos de los procesos sin y con membrana. Scielo, 28(3).

Barajas, D. J. R. H., \& Román, D. R. V. Simulación del proceso FCC: caracterización de las corrientes de alimentación y productos.

Cardoso Santos, L. J., \& Tenório, J. A. S. (2010). Avaliação do ciclo de vida e custeio do ciclo de vida de evaporadores para usinas de açúcar. Scielo, 63(1).

Ciro, H., Osorio, J. A., \& Cortés, E. A. (2008). Determinación del calor isostérico para pulpa de plátano (musa paradisiaca) por isotermas de sorción. Latindex, 75(156), 127-234.

Díaz-Ovalle, C. O., González-Alatorre, G., \& Alvarado, J. F. (2017). Analysis of the dynamic response of falling-film evaporators considering fouling. ScienceDirect.

Esteban, E., Saravia, L., \& Fernández, C. (2002). Producción de un destilador multietapa. ASADES, 03-19.

Ferreira, A., Egas, A., Domingues, A., Fernandes, C., \& Lobo, L. (2011). Vapor Pressure and Boiling Point Elevation of Eucalyptus Kraft Black Liquors. Scielo, 19(1)

Forero, L. E. P., Bernal, H. R. G., \& Guerrero, A. C. (2015). Efecto de las variables de evaporación: presión y flujo calórico en la calidad de la panela. Scielo, 16(1), 7-23.

Franco, J., Caso, R., Fernandez, C., Javi, V., \& Saravia, L. (2004). Aplicación de un concentrador tipo Fresnel para pasteurizar leche de cabra. Scopus, 57(8), 62-5. 
Greenfield, P. F., \& Geronimos, G. L. (1978). Effect of dextrans on the viscosity of sugar solutions and molasses. Evaluación de evaporadores industriales para jugo de caña. Scopus, 51, 67-72.

Hernandez, L. C., \& Borray, P. J. (17 de 07 de 2008). Retencio de nutrientes en bocadillos de guayaba y feijoa elaborados en evaporadores al vacio y presion atmosferica. Scielo, 6, 8.

Ibarz, A., \& Ribas, A. I. (2005). Operaciones unitarias en la ingeniería de alimentos. MundiPrensa Libros.

Josué R. Solís-Pacheco, F. P.-M.-Á.-M.-R.-R.-U. (17 de 01 de 2006). Descripción de un proceso tecnificado para la elaboración de piloncillo a partir de caña de azúcar. Latindex, 4, 9.

Montesano, J., \& Dipietro, A. Deshidratación de un alimento en cinta transportadora, programa para diseñar el proceso y evaluarlo económicamente.

Ordoñez, R. A., Hernández, C. A., \& Pedraza, L. F. (2012). Modelado de un sistema de evaporación de múltiple efecto para la producción de panela (azúcar no centrifugado). Scielo, 23(6), 105-120.

Paraíso, P. R., Andrade, C. M., \& Zemp, R. J. (2003). Destilação da miscela I: Modelagem e simulação da evaporação do hexano. Scielo, 23(3), 459-467.

Prada-Forero, L. E., Chaves-Guerrero, A., \& García-Bernal, H. R. (2015). Efectos de la presión de evaporación y la variedad de caña en la calidad de la miel y la panela. Scielo, 16(2), 153-165.

Prada Forero, L. E., García Bernal, H. R., \& Guerrero, A. C. (2014). Efectos de la presión de evaporación y la concentración de antiespumante y del uso de floculante y coadyuvante en la calidad de la miel y la panela. Scielo, 15(2).

Quintana, F. A. O., Maya, Y. L. I., Medrano, T. T., \& Montes, E. J. M. (2015). Efecto de la concentración y presión sobre la elevación del punto de ebullición de pasta de tomate (Lycopersicon esculentum Mill.). Scielo, 28(2), 51.

Rubio, L. A., Chernikoff, R. E., Muñoz, E., \& Sini, S. A. (2007). Estudio experimental para la predicción del funcionamiento de evaporadores. Scielo, 18(2), 83-86.

Sariego-Toledo, Y., García Noa, E., Montes de Oca, D., \& Guillén Rodríguez, C. (2014). Procedimiento general para la evaluación del proceso de remoción de calor en la elaboración de helados. Scielo, 34(3), 296-306.

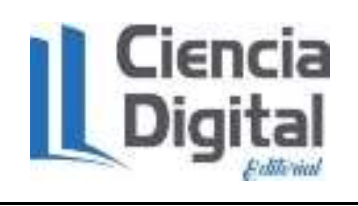




\section{PARA CITAR EL ARTÍCULO INDEXADO.}

Bonilla S., Acosta J., Miño G., Noguera A. \& Moreno M. (2019). Operaciones Unitarias: Enfocadas a los evaporadores en la elaboración de productos de consumo humano. Revista electrónica Ciencia Digital 3(2), 548-560. Recuperado desde: http://cienciadigital.org/revistacienciadigital2/index.php/CienciaDigital/article/view/451/1033

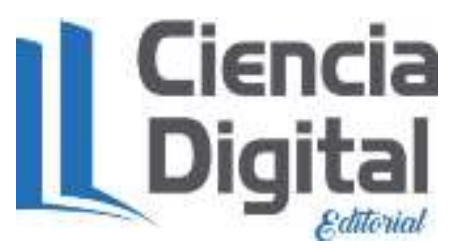

El artículo que se publica es de exclusiva responsabilidad de los autores y no necesariamente reflejan el pensamiento de la Revista Ciencia Digital.

El artículo queda en propiedad de la revista y, por tanto, su publicación parcial y/o total en otro medio tiene que ser autorizado por el director de la Revista Ciencia Digital.
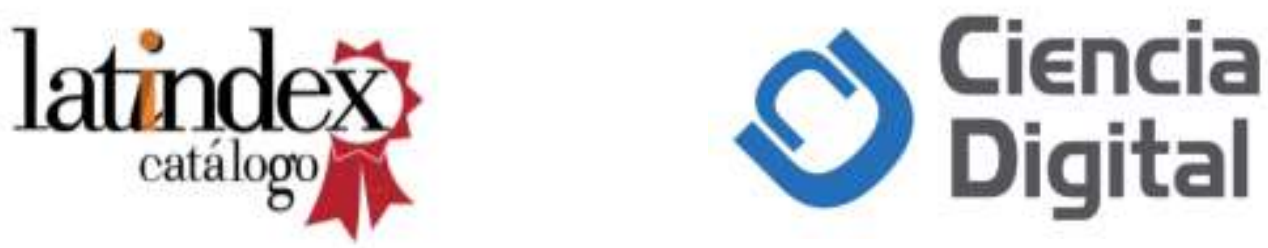